\title{
Restoration of the worn dentition
}

\author{
Paul King ${ }^{1}$
}

Received: 16 March 2017/Accepted: 31 May 2017/Published online: 30 June 2017

(C) The Author(s) 2017. This article is an open access publication

\begin{abstract}
Although tooth wear is considered to be age dependent, an increasing number of both adult and young patients is experiencing tooth wear, usually erosive in nature. It can present in variety of forms and severity. Prevention of further tooth wear should form the basis of care but in severe cases interventive restorative treatment may be necessary in order to protect vulnerable tooth surfaces and reestablish satisfactory appearance and function. Indications for interventive restorative treatment are: unacceptable appearance of the teeth, loss of normal function and progressive tooth wear resulting in pulp necrosis and/or difficulty in teeth restoration. The restorative treatment options possible with today's materials and techniques include: conventional fixed restorations, removable onlay/overlay prostheses and minimal preparation adhesive restoration. This article will review the restorative treatment options available to restore the worn dentition.
\end{abstract}

Keywords Worn dentition - Conventional fixed restorations · Removable prostheses - Minimal preparation adhesive restorations

\section{Quick reference/description}

Although tooth wear is considered to be age dependent, an increasing number of both adult and young patients are experiencing tooth wear, usually erosive in nature. It can present in a variety of forms and severity. Prevention of further tooth wear should form the basis of care, but in severe cases interventive restorative treatment

Paul King

paul.a.king@bristol.ac.uk

1 University of Bristol School of Oral and Dental Sciences, Lower Maudlin Street,

Bristol BS1 2LY, UK 
may be necessary to protect vulnerable tooth surfaces and re-establish satisfactory appearance and function. Indications for interventive restorative treatment are:

- Unacceptable appearance of the teeth

- Loss of normal function

- Progressive tooth wear resulting in pulp necrosis and/or difficulty in teeth restoration

The restorative treatment options possible with today's materials and techniques include:

- Conventional fixed restorations

- Removable onlay/overlay prostheses

- Minimal preparation adhesive restoration

\section{Overview (see Table 1)}

\section{Materials/instruments}

\section{Conventional fixed restorations}

- Porcelain-fused-to-metal (PFM) crowns

- All-metal crowns

- All-ceramic crowns

\section{Removable onlay/overlay prostheses}

- Acrylic resin

- Composite resin

- Cobalt-chromium framework

- Gold alloy framework

\section{Minimal preparation adhesive restorations}

- Composite resin

- Glass ionomer cements

- Resin modified glass ionomer cements

- Dentin bonding agent 


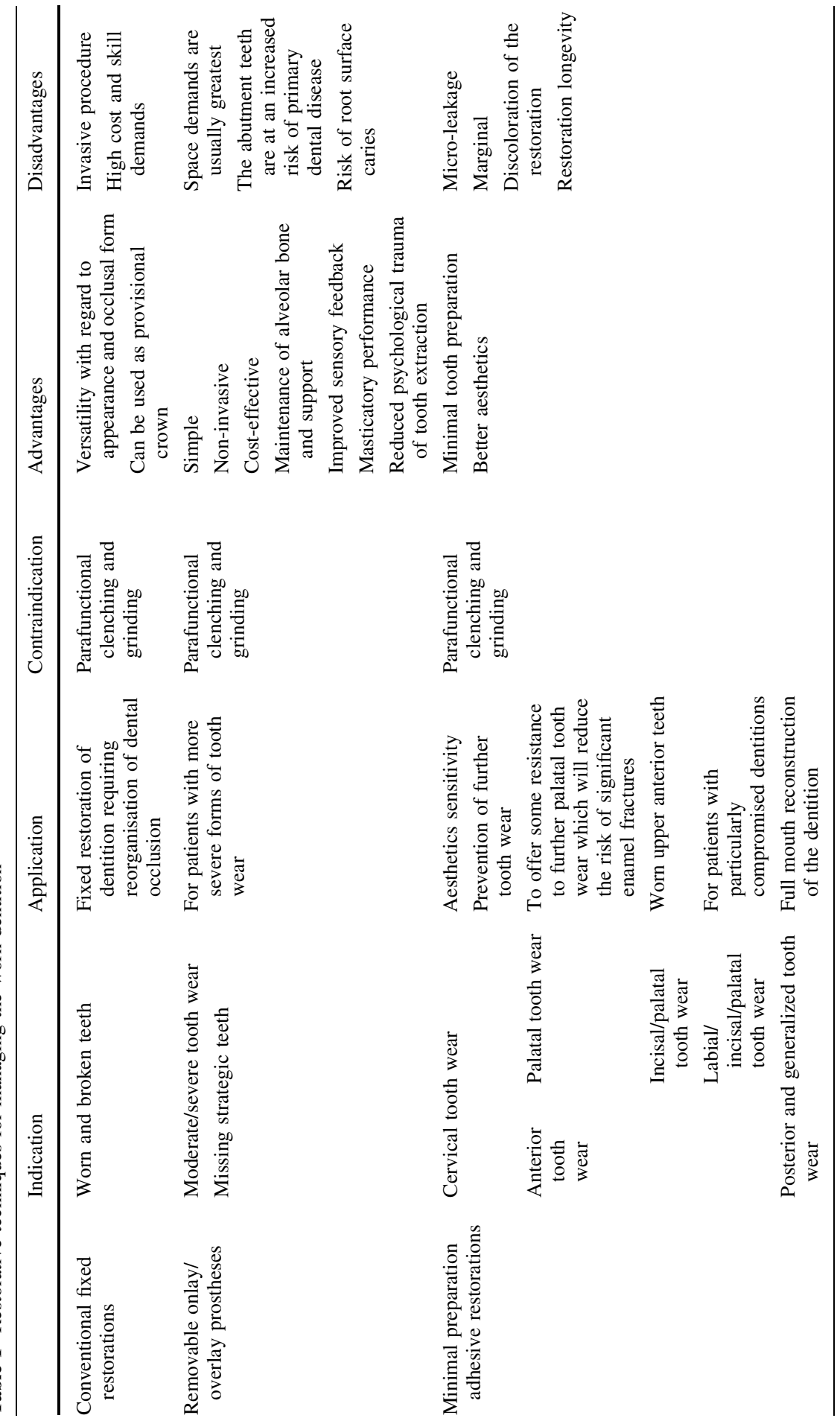


- Resin-bonded palatal metal alloy veneers (heat-treated gold alloys or nickelchromium alloys)

- Rubber dam

- Gingival retraction cord

- Modified porcelain laminate veneer

- Indirect densified composite resins

- Metal-ceramic crown

\section{Procedure}

\section{Conventional fixed restorations}

Conventional crown restorations, being an invasive procedure, require adequate interocclusal space which is usually lost as a result of compensatory eruption of opposing teeth during the process of tooth wear. Conventional restorative techniques to overcome the reduced crown height and lack of interocclusal space are:

- Opposing tooth reduction.

- Elective endodontic treatment and post retention.

- Occlusal adjustment (retruded arc of mandibular closure).

- Periodontal surgical crown lengthening (Fig. 1).

- Localized orthodontic tooth movement (conventional fixed appliance or 'Dahl' appliance) (Fig. 2).

- Overall increase in occlusal vertical dimension (Fig. 3).

\section{Removable onlay/overlay prosthesis}

It is recommended to construct a provisional acrylic resin removable prosthesis so that the shape, position, occlusal relationship of the prosthetic teeth and soft tissues as well as the patient's tolerance of a removable prosthesis can be assessed (Fig. 4).

The available space determines whether or not an anterior labial flange can be used or alternatively gingival fitting and/or butt-fitting tooth facings. The space demands are usually greatest in the anterior region both in the vertical and labiolingual dimensions (Fig. 5).

\section{Minimal preparation adhesive restorations}

Cervical tooth wear

Depending on the type of the lesion, different materials can be used (Fig. 6) (see Table 2). 

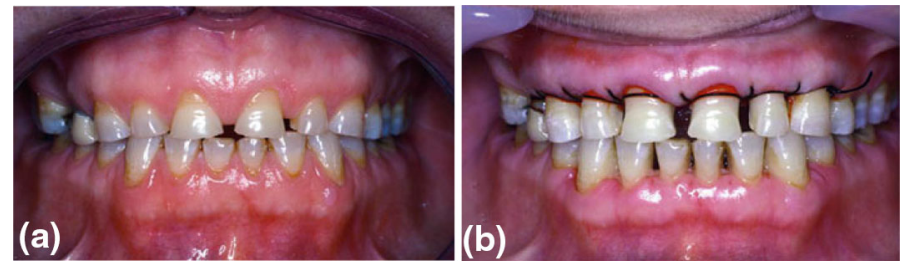

Fig. 1 Periodontal surgical crown lengthening on worn upper anterior teeth prior to the construction of conventional crown restorations. a Before surgery. b Immediately after surgery
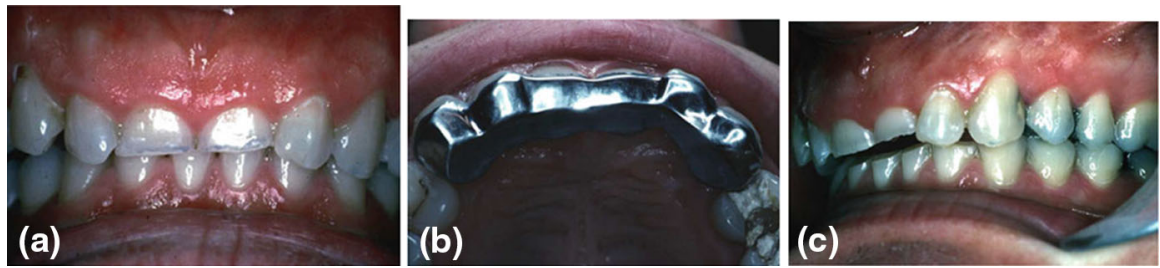

Fig. 2 a Localized anterior tooth wear. b 'Dahl appliance' cemented in place. c Interincisal space recreated following the removal of the 'Dahl appliance' prior to anterior restorations
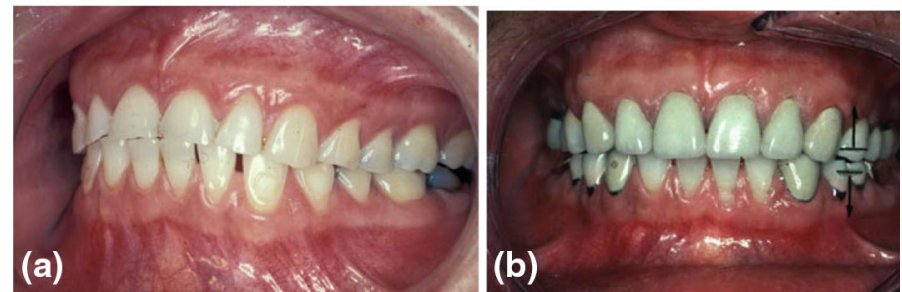

Fig. 3 Generalized tooth wear restored with conventional crown restorations in the anterior and posterior segments at an overall increase in occlusal vertical dimension. a Before restoration. b After restoration
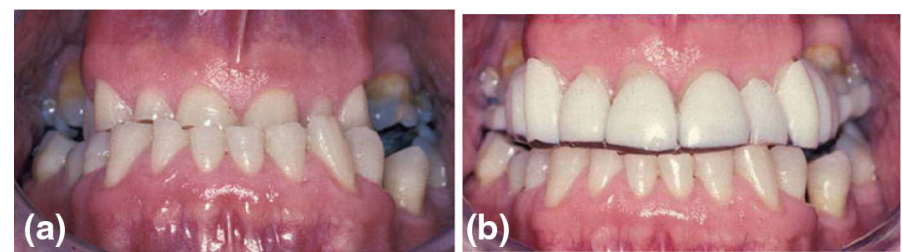

Fig. 4 Moderate/severe tooth wear with an unfavourable occlusal relationship initially restored with a provisional onlay/overlay removable prosthesis to assess appearance and function. a Before restoration. b After restoration with removable prosthesis

\section{Anterior tooth wear}

Palatal tooth wear To manage this form of tooth wear resin-bonded palatal metal alloy veneers can be considered. The incisal and palatal peripheral enamel margins are smoothened and laboratory fabrication of the metal alloy veneers is either done 

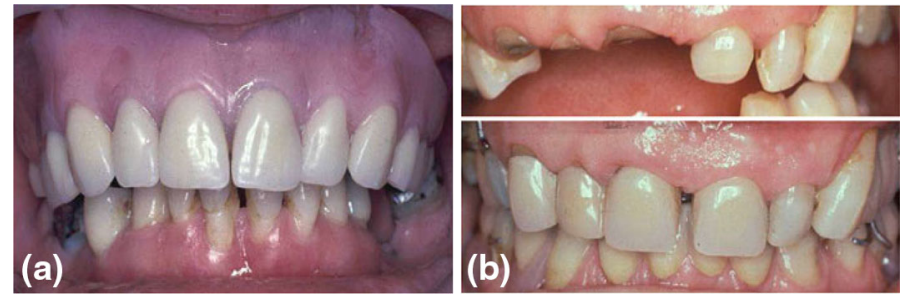

Fig. 5 a Full labial flange. b Gingival fitting anterior tooth facings
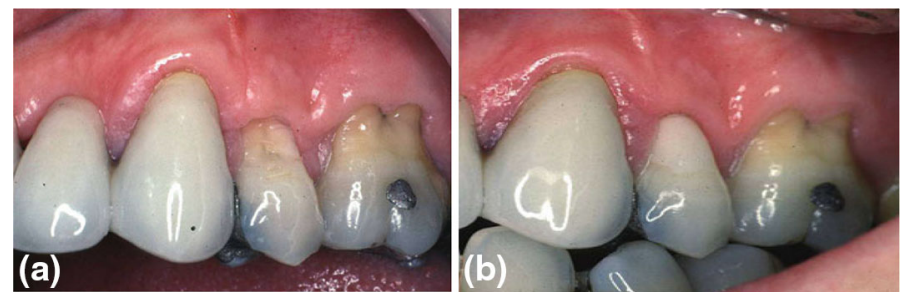

Fig. 6 Restoration of a cervical abrasion/erosion lesion using a layered glass ionomer and direct composite resin technique. a Before restoration. b After restoration

Table 2 Adhesive materials available for restoring cervical tooth wear

Lesions with margins that are still confined to enamel

Lesions that usually involve root cementum and dentin along with enamel

Higher aesthetic demand

Lesions are not visually prominent and involve more of the root surface, partly below the gingival margin

Deeper cervical lesions
Microfine or polishable densified composite resin, in conjunction with acid-etched enamel

Form of dentin bonding agent in combination with a composite resin or a self-adhesive composite resin formulation

OR

Glass ionomer cement

Polishable composite resin combined with some form of adhesive bonding agent

Glass ionomer cement

Glass ionomer cement and polishable composite resin

directly on a refractory working cast or by a wax/resin 'lift-off' technique. Interocclusal space is usually created to accommodate the thickness of the restoring material. When there is excessive tooth wear in the cervical region rubber dam isolation is used and occasionally gingival retraction cord. Cementation is done using luting cements that are usually resin-based and used in combination with the manufacturer's dentin bonding agent where appropriate (Fig. 7).

Incisal/palatal tooth wear The incisal portion of the tooth can be built with direct acid-etch retained composite resin and then a resin-bonded metal alloy palatal veneer can be constructed to cover both the palatal tooth tissue and composite resin by which the appearance of lost incisal and labial tooth tissue can be improved. 

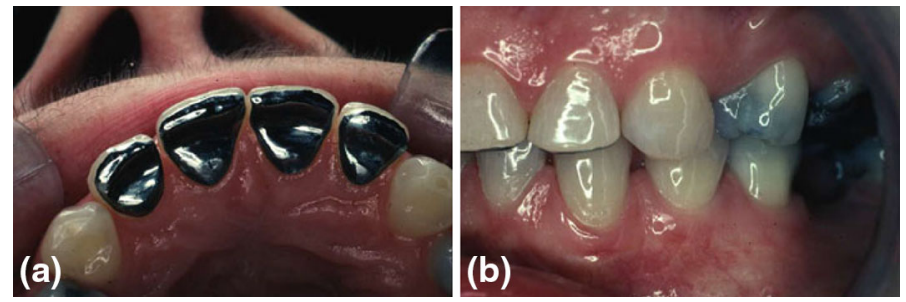

Fig. 7 Nickel-chromium alloy resin-bonded palatal veneers used to restore localized palatal tooth wear for maxillary incisor teeth. a Palatal view of veneers. b Labial view demonstrating re-establishment of posterior occlusal contacts

The incisal and palatal tooth surfaces can be restored conservatively with direct acid-etch retained composite resin at an increase in occlusal vertical dimension to accommodate the thickness of the restorative material.

Diagnostic wax-up is done on stone casts of planned restorations. Then rubber dam isolation of teeth is done prior to adhesive restorations. Silicone putty index and interproximal tape are used to aid restoration (Fig. 8).

A number of clinicians use modified porcelain laminate veneer restorations for the incisal and palatal worn tissue.

Indirect densified composite resins are the alternatives to using direct composite resins, with the potential advantages of improved physical properties and better control regarding occlusal and interproximal contouring.

Labial/incisal/palatal tooth wear All tooth surfaces can be restored with direct composite resin at an increased occlusal vertical dimension in an attempt to initially recreate lost interocclusal space. After that a decision can be taken either to continue with ongoing maintenance of the composite resin restorations or alternatively to consider proceeding to conventional crowns conforming to the newly established occlusion (Fig. 9).

In some cases with minimal tooth structure, localized crown lengthening surgery can be advantageous which will help to capture all remaining tooth enamel. If for any reason surgical crown lengthening is not available, then indirect splinted composite resin restorations can be considered to aid retention and durability (Fig. 10).

\section{Posterior and generalized tooth wear}

Resin-bonded heat-treated gold alloy restoration can be used in cases where aesthetics is not paramount (Fig. 11).

If aesthetics is a primary concern then a resin-bonded ceramic or indirect composite resin onlay can be considered.

In situations where retention and resistance form for conventional crowns are particularly compromised these techniques are helpful (Fig. 12).

In cases of generalized tooth wear, where a full mouth reconstruction of the dentition is indicated, the use of adhesive onlay restorations in the posterior quadrants can be considered in certain circumstances. If space is at a premium, the 

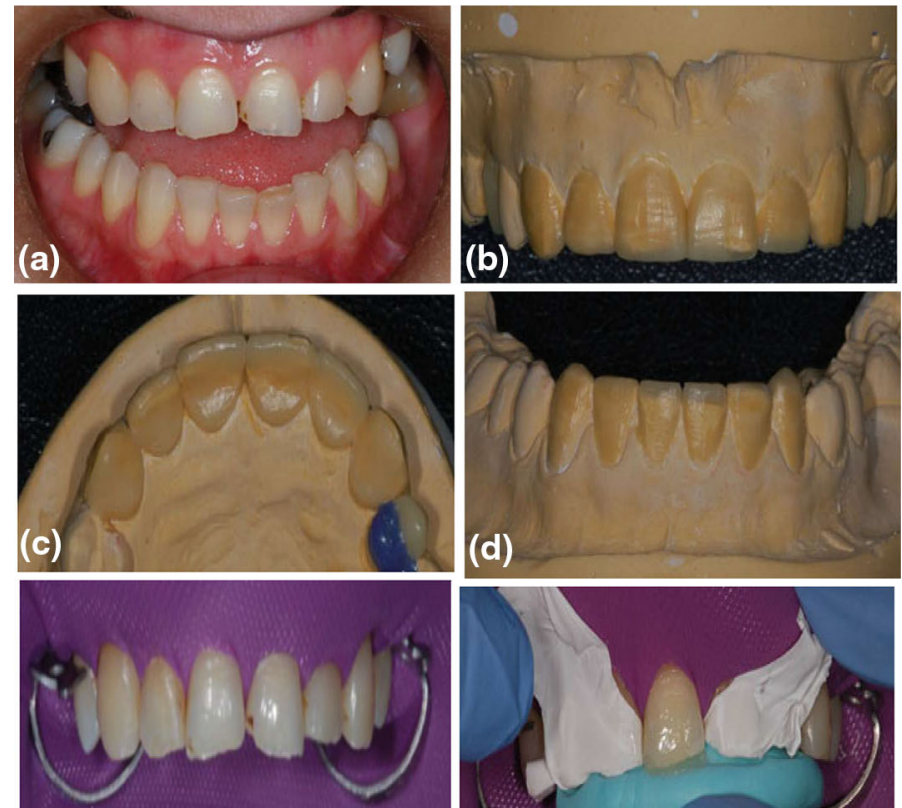

(e)
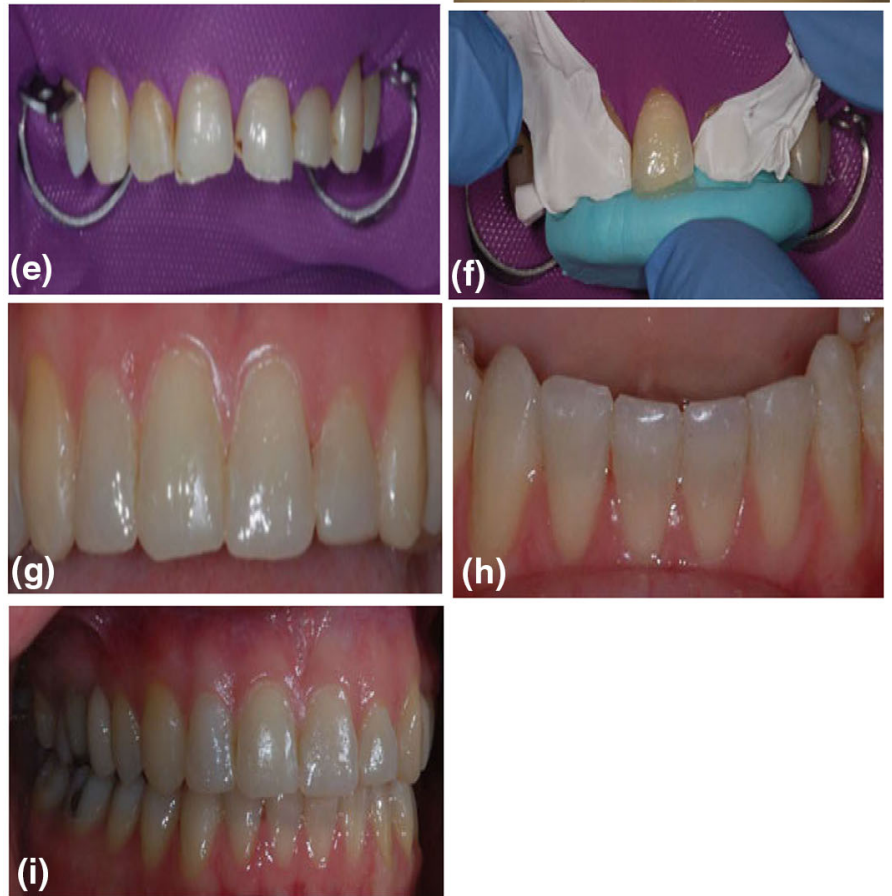

Fig. 8 a-i Restoration of worn upper and lower anterior teeth with direct composite resin restorations. a Before restoration. b-d Diagnostic wax-up on stone casts of planned restorations. e Rubber dam isolation of teeth prior to adhesive restorations. f Use of silicone putty index and interproximal tape to aid restoration. g Post restoration upper anterior teeth. h Post restoration lower anterior teeth. i Restorations 6 months following placement demonstrating re-establishment of posterior occlusal contacts (Illustrations courtesy of Dr. Tanya Cerajewska)

selection of a gold alloy instead of porcelain will be advantageous. In some cases a full mouth reconstruction of the worn dentition using resin-bonded ceramic or indirect composite resin restorations is possible. 

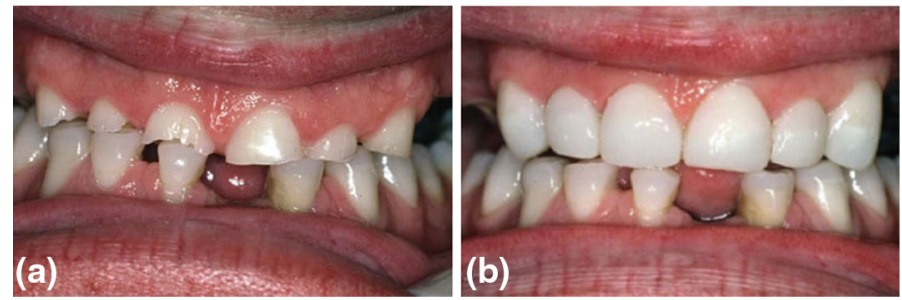

Fig. 9 Direct acid-etch retained composite resin restorations used to restore extensively worn maxillary anterior teeth at an increase in occlusal vertical dimension. a Before restoration. b After restoration
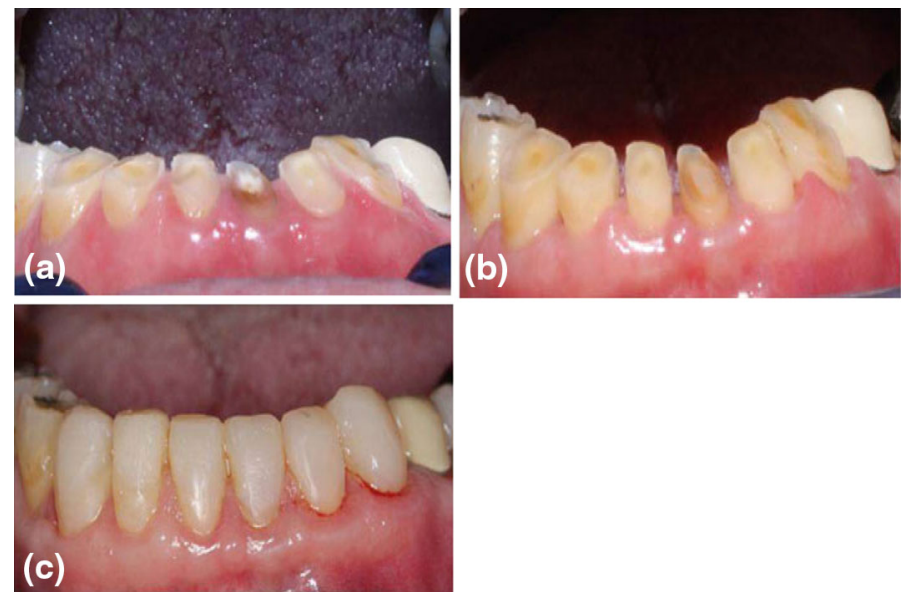

Fig. 10 Direct acid-etch retained composite resin restorations used to restore severely worn lower anterior teeth following periodontal surgical crown lengthening to expose available tooth structure. a Before surgery. b After periodontal surgical crown lengthening. c Immediately following placement of restorations (Illustrations courtesy of Dr. James Ban)

\section{Pitfalls and complications}

\section{Conventional fixed restorations}

- Pulp necrosis

- Tooth fracture

- Loss of cementation

- Marginal caries

- Postoperative sensitivity

- Interproximal spacing

- Placement of crown margins on root surfaces 

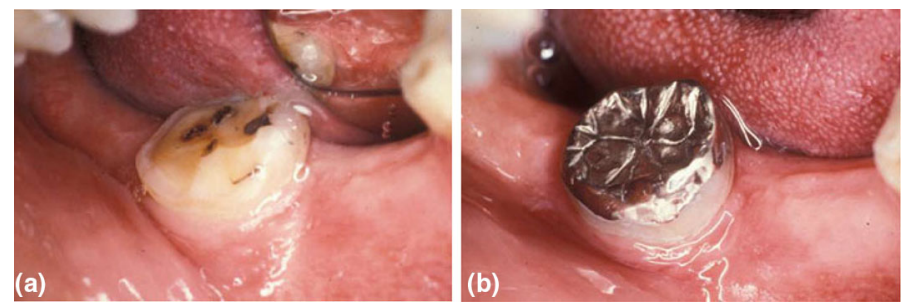

Fig. 11 Minimal preparation resin-bonded gold alloy onlay used to restore worn posterior tooth. a Before restoration. b After restoration

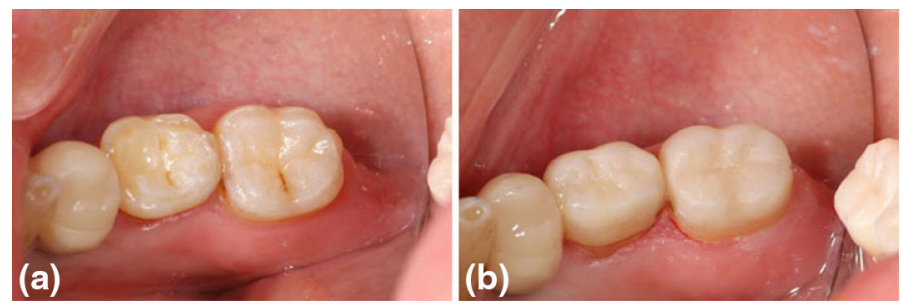

Fig. 12 Minimal preparation resin-bonded indirect composite resin onlay restorations used to restore worn posterior teeth. a Before restoration. b After restoration

\section{Removable onlay/overlay prostheses}

- Maintenance demands-material wear and fracture being common

- Difficulty in adapting both functionally and psychologically

\section{Minimal preparation adhesive restorations}

Unpredictable longer-term durability, particularly of the posterior onlay restorations; characteristically small fracture lines can appear in time which may eventually result in a catastrophic failure.

\section{Temporization}

Procedures involving complete resin bonding of the temporary restoration to the underlying tooth tissue may compromise the subsequent adhesive bond for the final restoration.

\section{Damage to tooth structure}

Risk of damage to the tooth preparation during the removal of the interim resin lute. 


\section{Adhesive material}

Using a less adhesive material or technique can result in the early loss of any temporary restorations, with the possible consequences of unplanned tooth movement. In try-in stage checking the occlusal relationship can be a challenge due to the relative lack of retention of the restorations before cementation.

Open Access This article is distributed under the terms of the Creative Commons Attribution 4.0 International License (http://creativecommons.org/licenses/by/4.0/), which permits unrestricted use, distribution, and reproduction in any medium, provided you give appropriate credit to the original author(s) and the source, provide a link to the Creative Commons license, and indicate if changes were made.

\section{References}

1. King PA (2015) Restoration of the worn dentition. In: Amaechi BT (ed) Dental erosion and its clinical management. doi:10.1007/978-3-319-13993-7_13

2. Mehta S, Banerji S, Millar B, Suarez-Feito J (2012) Current concepts on the management of tooth wear: part 1. Assessment, treatment planning and strategies for the prevention and the passive management of tooth wear. Br Dent J 212:17-27

3. Mehta S, Banerji S, Millar B, Suarez-Feito J (2012) Current concepts on the management of tooth wear: part 2. Active restorative care 1: the management of localised tooth wear. Br Dent J 212:73-82

4. Mehta S, Banerji S, Millar B, Suarez-Feito J (2012) Current concepts on the management of tooth wear: part 4. An overview of the restorative techniques and dental materials commonly applied for the management of tooth wear. Br Dent J 212:169-177 